\title{
FLOW SHARING SYSTEMS FOR MOBILE APPLICATIONS
}

\author{
Torben Ole ANDERSEN*, Michael Rygaard HANSEN** and Finn CONRAD*** \\ *Institute of Energy Technology, Section of Fluid Power and Mechatronic Systems \\ Aalborg University, Pontoppidanstraede 101, Aalborg, DK-9220, Denmark \\ (E-mail: toa@iet.auc.dk) \\ ** Institute of Mechanical Engineering, Aalborg University, Section of Computer Aided Design \\ Pontoppidanstraede 101, Aalborg, DK-9220, Denmark \\ ***Department of Mechanical Engineering, Section for Engineering Design and Product \\ Development, Technical University of Denmark, DK-2800, Kgs. Lyngby, Denmark
}

\begin{abstract}
This contribution reports about some analytical and simulation/experimental studies carried out on different flow control systems for mobile applications with respect to their ability to do flow sharing. All systems have two parallel actuators and are considered regarding functionality and complexity. The aim is to compare well established systems/methods and systems involving electronic sensor technology.
\end{abstract}

\section{KEY WORDS}

Flow sharing, Mobile valves, Load sensing

\section{INTRODUCTION}

In the most common types of mobile valves the proportionality between the main spool travel and the flow of each service is only valid as long as the proportional valve is unsaturated, i.e., the sum of the flow demand of each activated service lies below the available pump flow. Typically, the service with the highest load pressure will stall before any reduction in flow is experienced be the lesser loaded services [1].

In Figure 1 is schematically shown a load sensing (LS) load independent directional control valve group consisting of two pressure compensated orifices and a pressure adjustment spool.

When the main spools of the individual modules are closed, all the flow from the pump is led across the

\begin{abstract}
pressure adjustment spool to tank. The spring and flow force determines the stand-by pressure. When one or more of the main spools are activated, the pump pressure is determined by the highest load pressure.

This is done either via the pressure adjustment spool, through the shuttle valve circuit, as shown in Figure 1 (fixed displacement pumps) or via a pump regulator (variable displacement pumps).

n this way the pump pressure will always correspond to the load pressure plus the stand-by pressure of the pressure adjustment spool or the variable pump. The modules utilise their internal pressure compensators to ensure that the pump flow is distributed as required.

Consider a valve containing 2 activated services with locked main spools and differently loaded (1: lower pressure, 2: higher pressure).
\end{abstract}




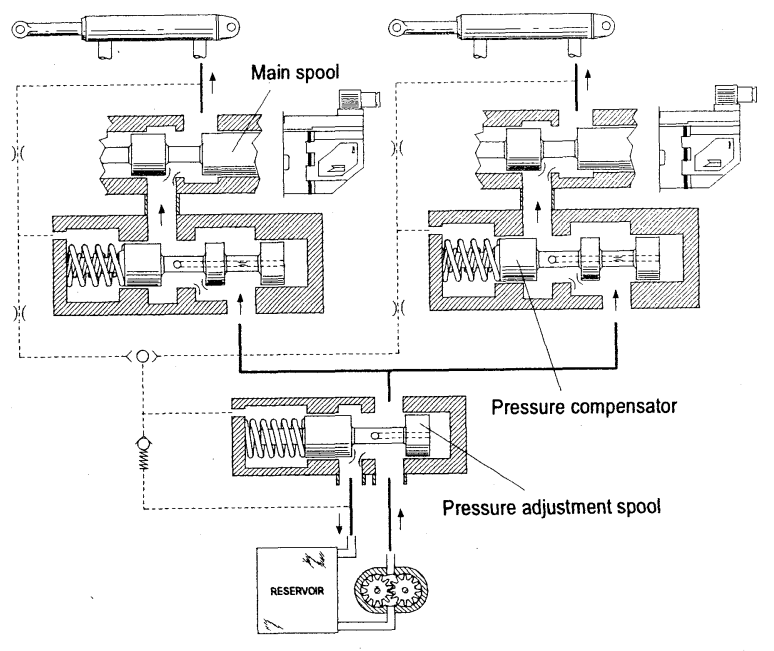

Figure 1 Load sensing load independent directional control valve group, with 2 services.

In Figure 2 the flow across the pressure adjustment spool and the service flows are plotted as function of the pump flow.

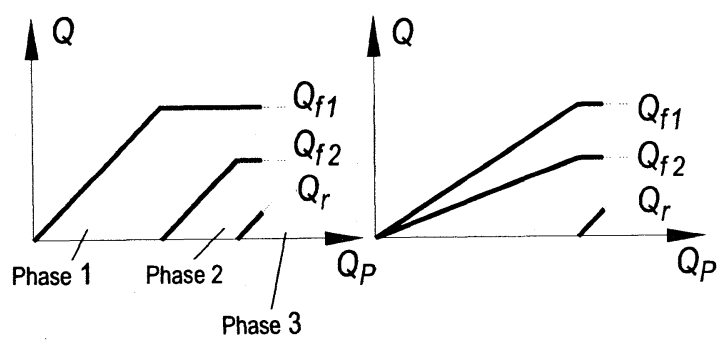

Figure 2 Return, $Q_{r}$, and service flows, $Q_{f 1}$ and $Q_{f 2}$, vs. pump flow, $Q_{p}$ for 2 valve groups.

The plot to the left corresponds to a typical mobile valve and represents a $100 \%$ absence of flow sharing. Introducing the term pump-LS pressure margin as the difference in pump and LS pressure the behaviour may be divided into 3 phases:

1. The pump-LS pressure margin is negative. All the flow is directed to service 1 . The pressure adjustment spool is held closed. The pressure compensator of service 2 is moved so far to the right, see Figure 1, that its build in contra valve function is activated closing off the connection between the pump and the main spool.

2. The pump-LS pressure margin is positive but less than the crack pressure of the spring of the pressure adjustment spool. The flow to service 1 is pressure compensated at $Q_{f 1}$ and the remaining flow is directed to service 2. The pressure adjustment spool remains closed.

3. The pump-LS pressure margin is larger than the crack pressure of the spring of the pressure adjustment spool.
Both service flows are pressure compensated at $Q_{f 1}$ and $Q_{f 2}$, respectively. The remaining flow goes across the pressure adjustment spool, i.e., there is no saturation in the system.

The saturation associated with especially phase 1 but also phase 2 typically reduces manoeuvreability and jeopardises safety during operation. Hence, it is considered a major competition parameter for an LS directional valve. The desired flow pattern corresponding to optimal flow sharing is shown in Figure 2, to the right.

The aim of this paper is to describe different mechanical and electrical solutions to this problem.

\section{MECHANICAL SOLUTIONS}

A method used in commercial valves to overcome this saturation problem is shown in Figure 3.

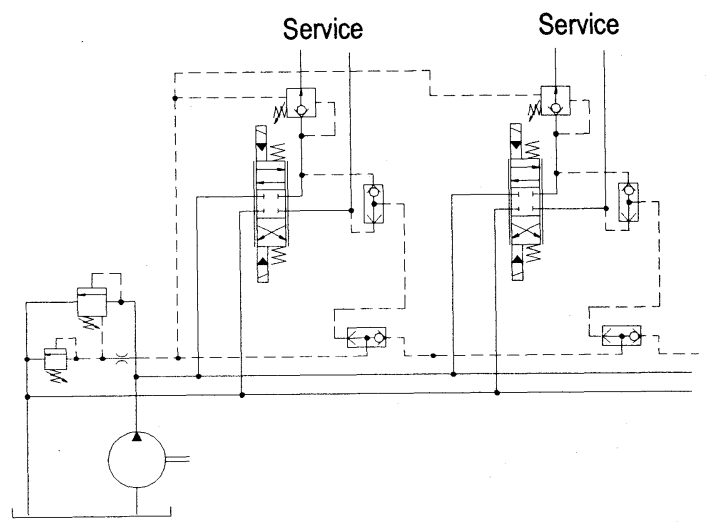

Figure 3 Hydraulic circuit of LS directional control valve offering flow sharing.

The pressure compensators are positioned downstream of the metering spools and are all acted upon in the closing direction by the LS pressure. Thereby, a seemingly constant pressure drop is generated across the main spools and the pump-LS pressure margin is forced positive at all times.

There are, however, drawbacks associated with such a solution:

- A more complicated metering spool and compensator combination is required.

- A certain loss of flow control is experienced as the pressure drop across the metering spools are directly affected by the slope of the $\triangle \mathrm{P}-\mathrm{Q}$ curve of the pressure adjustment spool.

Figure 4 clearly indicates the second of the above mentioned drawbacks.

The steady state simulations are carried out on for a fixed loading condition:

- 1 st unit: 80 bar - $20 \mathrm{l} / \mathrm{min}$

- 2nd unit: 300 bar - $10 \mathrm{l} / \mathrm{min}$ 
Thus, the LS signal of the valve will be 300 bar and flow sharing and/or saturation appears for pump flows below $30 \mathrm{l} / \mathrm{min}$.

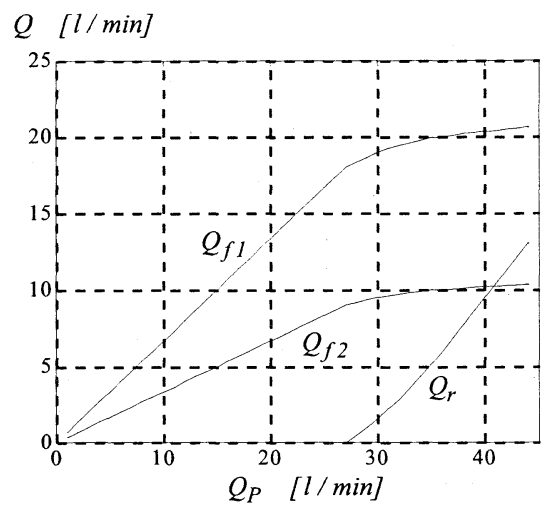

Figure 4 Flow pattern of the valve in Figure 3

The loss of flow control seen in Figure 4 can be eliminated by adding an extra compensator as shown in Figure 5 and 6.

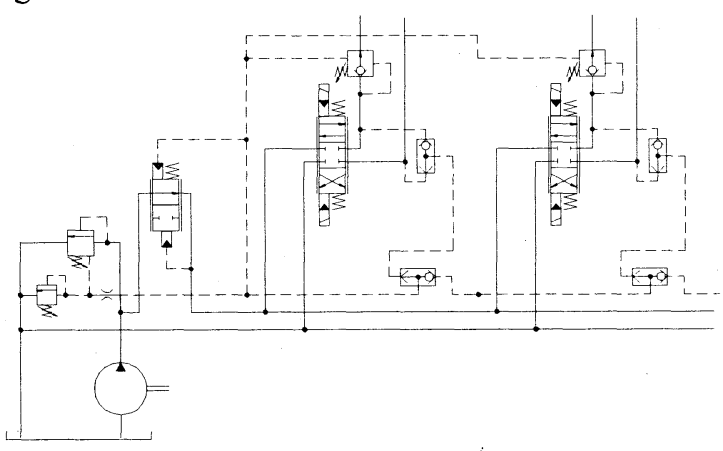

Figure 5 Hydraulic circuit of LS directional control valve offering flow sharing.

This will, however, increase costs and reduce stability margins for the valve. Maintaining the pressure compensator upstream of the metering spool offers a number of advantages.

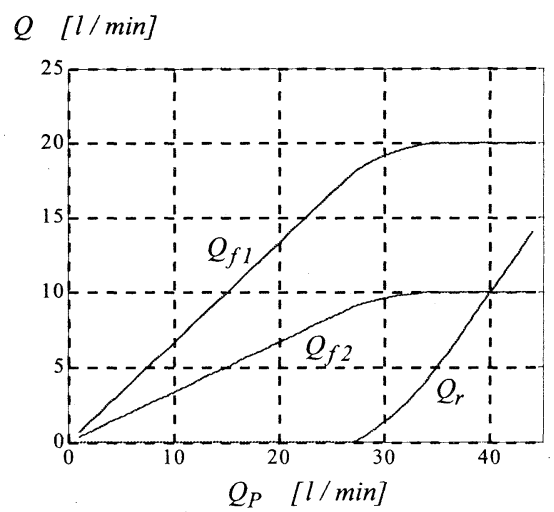

Figure 6 Flow pattern of the valve in Figure 5
First of all, only one compensator is required pr. service but also, existing valve configurations may be maintained, i.e., costs related to change in production may be avoided or at least reduced. For this type of valve, the main task when designing a configuration that will offer flow sharing is to keep the pump pressure above the LS pressure at all times. As described earlier, the pump-LS pressure margin directly indicates which of the 3 phases the valve is experiencing, i.e., a mechanical solution not depending on any sensors would have to be based on this margin.

Naturally, this can be done in several ways, however, the set up will typically be complex and require some tuning. In Figure 7 an example of such a system is shown.

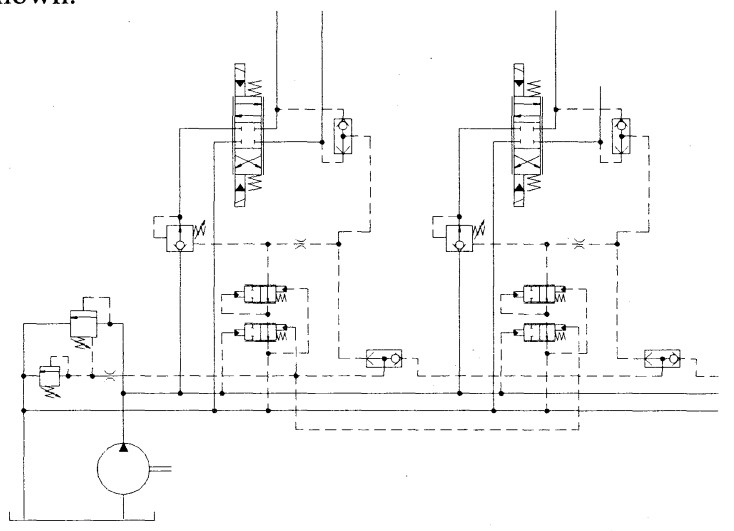

Figure 7 Hydraulic circuit of LS directional control valve offering flow sharing.

Each service is supplied with an adjustable orifice that depends on the pump-LS pressure margin such that it is fully closed when the valve is in phase 3 , and fully open when the margin is zero.

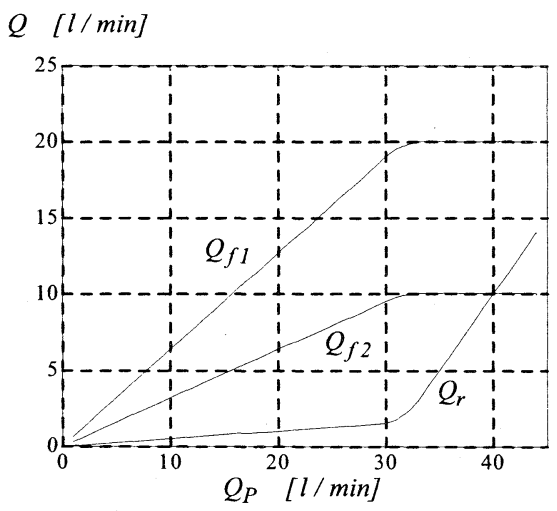

Figure 8 Flow pattern of the valve in Figure 7

Also each service contains a compensator that maintains constant pressure drop across the adjustable orifice. Together with a fixed orifice the set up bleeds off the service pressure chamber of the service compensator. 
By careful tuning of orifice and spring data this will lead to a system where the pressure compensators closes as the pump-LS pressure margin goes below the crack pressure of the spring of the pressure adjustment spool. Thereby the pressure adjustment spool will never fully close, and the pump-LS pressure margin is preserved for any level of pump flow. In Figure 8 the flow pattern is shown. Beside the obvious disadvantage of the increase in moving parts as well as the required tuning, a major drawback is the loss in volumetric efficiency when flow sharing. This is mostly caused by the flow across the pressure adjustment spool.

\section{ELECTRICAL SOLUTIONS}

To simplify the operators work coordinated or resolved motion control systems are becoming more and more interesting [2]. These systems involves in general calculation of the inverse kinematics to determine the necessary angular adjustment of the individual link to obtain the desired tool centre point movement. In these systems it is necessary to have a system for proportioning the fluid flow to the various actuators, when the demanded flow exceeds the available flow, so that only the speed is decreased and not the direction. These systems involve natural electro-hydraulic actuated valves.

One basic scheme operates with an adjusted reference signal for the spool travel of the main spools that deviates from the user reference signal when saturation is sensed: If the pump-LS pressure margin is less than the crack pressure of the spring of the pressure adjustment spool the adjusted reference signal for any activated metering spool should be moved towards its dead band value. If the pump-LS pressure margin is more than the crack pressure of the spring of the pressure adjustment spool the adjusted reference signal for any activated metering spool should be moved towards its original user defined value.

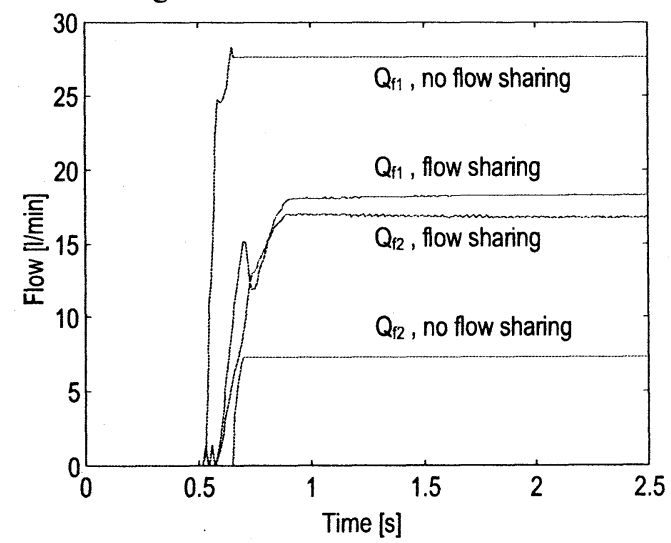

Figure 9 Service flows for an LS directional control with and without flow sharing control scheme
In Figure 9 the flow pattern of the system is shown as response to a step input in the reference signal to both main spools of a dynamic valve model.

The flow patterns clearly show the beneficial effect of the implemented flow sharing scheme. This method is sensitive to the discharge area characteristics of the different main spools since it controls the spool travel rather than the discharge area. In general, the discharge flow characteristic of main spools are either linear or slightly progressive. In this case 2 identical metering spools were modelled, hence, perfect flow sharing that would divide the flow in exact halves could have been expected, however the difference in load pressure can be recognized.

For electro-hydraulic systems, in general, an often used method involve measuring the flow delivered by the pump, either by direct flow measurement or calculation from displacement and speed of the pump. With this information the movement of each link is reduced when the demanded flow exceeds pump capacity.

To make these systems effective it is necessary to know the demanded flow. Using a pressure compensated valve, it is possible to tabulate the relation between input signal and output flow, thereby having an open loop system.

More modern valves utilise pressure transducers to measure the pressure drop across the metering orifices, making it possible to calculate the flow, via the well known orifice equation. This gives a direct flow measurement and the input signal can be adjusted to cope with machine capacity. This solution, however, involves a feedback loop, and analysis is needed to assure global stability.

\section{CONCLUSIONS}

The current paper examines a number of different mechanical and electrical solutions to the problems of flow saturations experienced by typical mobile valves. Mechanical solutions exist that solves some of the basic problems related to maneoeuvreability and safety. However, the increasing demands to functionality and motion control, such as resolved or coordinated motion control, clearly points toward electrical solutions.

\section{REFERENCES}

1. Hansen, M. R. Andersen, T. O., Improved functionality and Performance of Mobile Cranes Using Pressure Feedback, London Drives and Controls 2001, 6th Annual exhibition and Conference.

2. Sepehri, N., Lawrence, P.D., Sassani, F. and Frenette, R. Resolved-Mode Teleoperated Control of Heavy-Duty Hydraulic Machines. Trans. ASME, Journal of Dynamic Systems, Measurements and Control, 1994, 166, pp. 232-240. 